\title{
Construcción de un instrumento para conocer las condiciones laborales de los artistas peruanos
}

\author{
Construction of a tool to know the working conditions of the Peruvian artists
}

\author{
Jorge Luis Yangali Vargas 1 \\ Juan Reymundo Vega ${ }^{2}$ \\ Rusel Hilario Daga Salazar ${ }^{3}$ \\ Adolfo Ramos Aliaga 4 \\ Gina Mayta Salomés
}

RECIBIDO: 9 DE ABRIL DE 2020

ACEPTADO: 5 DE JUNIO DE 2020

\section{RESUMEN}

El presente artículo corto es preliminar del proyecto de investigación que consiste en estudiar las condiciones laborales de los artistas peruanos. Nuestro objetivo es presentar a la comunidad académica la construcción de una guía de encuesta que

\footnotetext{
' Doctor en Letras Modernas, Licenciado en Pedagogía y Humanidades, Docente, Universidad Nacional del Centro del Perú, Perú; jyangali@uncp.edu.pe, ORCID: https://orcid.org/0000-0003-3714-326X

Google Scholar: https://scholar.google.com.pe/citations?user=GgiAvrMAAAAJ\&hl=es

2 Licenciado en Educación, Docente, Universidad Nacional del Centro del Perú, Perú, wankanuna@yahoo.es. ORCID: https://orcid.org/0000-0003-3875-189X

3 Ingeniero de Sistemas, Administrativo en la Universidad Nacional del Centro del Perú, Perú, Perú, ruseldaga@uncp.edu.pe, ORCID: https://orcid.org/0000-0003-3636-5602.

Google Scholar: https://scholar.google.com/citations?hl=es\&user=DOQI7EQAAAAJ

${ }_{4}$ Maestro en Artes, Artista Plástico, Docente, Universidad Privada Los Andes, Perú, ramos_aliaga@hotmail.com, ORCID: https://orcid.org/0000-0002-4302-7460

Google Scholar: https://scholar.google.com/citations?hl=es\&authuser=2\&user=ajg4Z_4AAAAJ

5 Doctora en Educación, Licenciada en Educación, Docente del Ministerio de Educación, Perú, elianasm_20@hotmail.com, ORCID: https://orcid.org/0000-0003-4224-9958
}

Socialium revista científica de Ciencias Sociales, Vol 4 - No. 2, julio - diciembre 2020, pág. 281-298.

DOI https://doi.org/10.26490/uncp.sl.2020.4.2.678 


\section{2}

permita ahondar en la problemática laboral de los mismos. Metodológicamente se siguieron los pasos precisados por la metodología científica; esto es la validación del contenido mediante juicio de expertos y la aplicación de una prueba piloto que permitió determinar un valor de confiabilidad de $a=0.911$ de los ítems formulados en este instrumento. La guía de encuesta combina preguntas abiertas y cerradas con la finalidad de recabar además de datos cuantitativos, narrativas de la experiencia laboral.

Palabras clave: condiciones de trabajo, método de investigación, trabajo de artista, encuesta, Perú

\section{ABSTRACT}

This short paper is a preliminary to the research Project; That consists in studying the working conditions of Peruvian artists. Our objective in the article is present to academic community the construction of a survey guide that will allow them to delve into their employment problems. Methodologically, we continue the steps specified by the scientific methodology; This is the validation of the content through expert judgment and the application of a pilot test that determines a reliability value of $a=$ 0.911 for the items formulated in this instrument. The survey guide combines open and closed questions in order to collect. The purpose of gathering, in addition to quantitative data, narratives of work experience.

Keywords: working conditions, scientific methods, works of art, surveys, Peru

\section{Introducción}

El 28 de julio de 2020 se cumplirán más de quince años de la vigencia de la Ley del Artista, Ley 28131 (2003) y su respectivo Reglamento (2004). Se trató de una ley que emergió después de diez años de aplicación del modelo económico neoliberal. De algún modo su promulgación fue en respuesta a las demandas globales de organismos como la Organización Internacional del Trabajo; pero también fue producto de la presión interna de colectivos artísticos que participaron activa, creativa y decisivamente contra la dictadura fujimorista. Años después, 20 de julio de 
2010, sería creado el Ministerio de Cultura, que cuenta con una Dirección General de Industrias Culturales y Artes, la misma que depende del Viceministerio de Patrimonio Cultural e Industrias Culturales. Como se ve, jurídica e institucionalmente el Perú en lo que a la cultura y arte se refiere se ha organizado y regulado de modo que la sociedad, en especial, la clase política, viene mostrando su interés por incluir a las artes y con ellas a los artistas en política públicas.

Desde la creación del Ministerio de Cultura una de sus tareas más significativas ha sido el levantamiento del patrimonio cultural (material e inmaterial) nacional, lo cual ha influido en la percepción ciudadana respecto de importancia de reconocer y promover la diversidad de las manifestaciones artísticas colectivas e individuales. Otro de sus ejes de trabajo ha sido la creación y administración de fondos que alientan la práctica de las artes en general; por ejemplo, el (2019) el Ministerio de Cultura ha dispuesto más de medio millón de dólares para incentivarlas, mediante el sistema de concurso. Se trata de experiencias significativas en cuanto al mecenazgo de parte del Estado peruano para con sus ciudadanos artistas. En ese sentido el Ministerio de Cultura (2016) sigue los lineamientos que la UNESCO propone en lo relativo a la condición del Artista.

De cara al bicentenario, este contexto que nos recuerda el Decreto que firmara José de San Martín respecto de las prácticas teatrales, resarciéndolas de una serie de infamias que les atribuían tanto a quienes las practicaban como a quienes se "contaminaban" yendo a ver las obras. Entre las muchas injurias que se hacían y que aún persisten en el imaginario popular, aluden a las condiciones laborales y morales de sus oficiantes, creyéndose el oficio del artista como oficio de vagos y pervertidos. El año 2006 junto a Adolfo Ramos elaboramos la Sinopsis de la producción de los pintores huancaínos. En dicho estudio pudimos elaborar un directorio de los artistas plásticos. También describimos sus referentes como artistas; esto es sus exposiciones, grupales e individuales, su formación y estudios, sus estancias en el extranjero, etc. También se eligió una de sus obras, la que el mismo artista consideraba más representativa de su producción, para realizar un análisis técnico de la misma: técnica, tendencia, medidas, materiales empleados, etc. Este estudio seminal no tuvo continuidad. Registramos en actividad a 49 artistas en Junín. 


\section{4}

El 2018 (Yangali, 2019) volvimos nuevamente nuestro interés hacia la problemática del artista, en dicha ocasión hacia su formación en el marco jurídico que homogenizó la labor de las instituciones de formación artística con la labor universitaria y las exigencias que esto implica: la exigencia de los grados de Maestro y Doctor a los docentes de estas instituciones, la modalidad de titulación mediante la elaboración de una tesis, etc.

La preocupación por las condiciones del artista latinoamericano, como se puede comprobar en la escasa bibliografía al respecto no ha sido de interés público ni académico en muchos lustros. No obstante, en los años recientes hay un creciente interés. Se tienen dos grupos académicos; uno en México y otro en Argentina que vienen asumiendo el reto de generar la reflexión al respecto. En México se tiene al equipo alrededor de la Revista Latinoamericana de Antropología del Trabajo (2020, de próxima edición). Y en Argentina en torno a la revista Telón de Fondo (Varios 2018). Ambos equipos han optado por al exploración documentaria e histórica, en especial el equipo dirigido por Karina Mauro (2018). De ahí que en lo metodológico aún hay muchos terrenos desde donde se puede explorar la problemática. Motivo por el cual nuestro objetivo fue construir un instrumento que nos permita obtener datos tanto cuantitativos como cualitativos de la problemática laboral del artista peruano y en particular del de Junín.

\section{Método}

La investigación es de tipo básica con diseño básico descriptivo. Luego de hacer una revisión exhaustiva de las bases de datos de acceso abierto nacionales como Alicia de Concytec, así como de los multidisciplinares Scielo y Redalyc se constató que no se cuenta con fuentes especializadas en los artistas. Siendo los sectores laborales más estudiados los vinculados a la agricultura, educación y salud. En tal sentido, para la construcción de nuestra encuesta se tomaron en cuenta los aportes de Myriam Ganoza (1986), Remedios Raqueta (1995), Ricardo Cuenca (2006), Magaly Robalino (2006) y Custodio Arias (2009).

El instrumento fue sometido a validación de contenido entre un equipo multidisciplinario y multinacional de expertos. Las filiaciones institucionales de los seis expertos son las siguientes: Universidad de Buenos Aires, Universidade do Vale do 
Sapucaí - UNIVÁS, Universidade Federal do Rio Grande do Sul, Universidad Nacional del Centro del Perú, Universidad Privada los Andes, Universidad Continental. Las líneas de investigación de los mismos tienen que ver con la Investigación social y artística (02), investigación educativa (04), investigación laboral (02), investigación psicológica (02), investigación de las industrias culturales (02).

El mecanismo de validación consistió en enviarles por correo la primera versión, luego realizarlas mejoras en función a las sugerencias y finalmente retornar la versión mejorada del instrumento.

Las observaciones de los expertos nos permitieron realizar correcciones en la especificidad de las preguntas. Por ejemplo, en las condiciones de salud se añadió un ítem para saber si ante uno de los problemas físicos, mentales y psicológicos el artista recibía atención y tratamiento médico.

Luego de la validación, por una cuestión metodológica y por sugerencia de los expertos se llevó a cabo una prueba piloto. Para lo cual se trabajó con los artistas plásticos del valle del Mantaro. Se aplicó la encuesta a 17 artistas. Los mismos que participaron previo consentimiento informado (véase el segundo anexo).

\section{Resultados}

Los indicadores de la encuesta los hemos distribuido en las siguientes secciones: Los datos generales tienen la intención de generar un perfil socio familiar del artista. En las secciones Formación Académica y Condiciones de Salud procuramos ahondar tanto en la formación vocacional y educativa; como sobre sus condiciones de salud y conocer así sus principales padecimientos ergonómicos y mentales así como a los agentes que velan por su atención médica y ulterior jubilación.

Como es de esperarse por la naturaleza de nuestra investigación la sección central son las Condiciones Laborales. Se recogen datos sobre tiempo dedicado al arte en el entendimiento de este como trabajo remunerado; asimismo se recogen datos sobre dedicación a la capacitación y tiempo de ocio o recreación teniendo como referencia una jornada diaria o semanal.

En la sección Condiciones materiales de trabajo artístico pedimos al entrevistado una descripción del lugar de trabajo artístico, los mecanismos la provisión de equipos, 


\section{6}

materiales e insumos; y el vínculo que tiene con sus "empleadores", y otras organizaciones gremiales.

Las dos últimas secciones, Experiencias laborales y Actitud frente a la labor artística, tienen la intención de ahondar en primer lugar en sus narrativas laborales sobre sucesos específicos como despido, ascenso, etc. En segundo lugar, se le pide al artista un ejercicio epistemocrítico para definir en sus propios términos su labor como trabajador y grado de satisfacción sobre el mismo.

Considerando la naturaleza de las investigaciones de corte cualitativo y cuantitativo (mixto), con una postura socioeconómica seguimos la postura de Álvarez (2003:43) respecto de los estudios piloto. Aplicamos la prueba piloto para conocer el grado de confiablidad de los ítems de la encuesta. Inicialmente se ha aplicado un Guía de encuesta con preguntas tanto abiertas como cerradas. Asumimos que el mecanismo de este instrumento es el más adecuado para determinar las modalidades de las variables que podrían utilizarse en el cuestionario final (ver anexo 1). Para el tratamiento estadístico, todas las preguntas abiertas se han tratado de cerrarlas asignándoles escalas a fin de homogenizar a los ítems.

De acuerdo a Menéndez (2016, p. 79) el coeficiente alfa de Cronbach "se basa en el cálculo de la confiabilidad de un compuesto donde cada ítem se considera un subcuestionario del cuestionario total y los ítemes se consideran cuestionarios paralelos". Por lo que diseñamos las escalas para cada pregunta (o ítem) de la Guía de encuesta con los descriptores que hacen conocer las condiciones laborales del artista en Junín, dicha encuesta resultó constituida por 86 ítems en una hoja, "Sistematización", en el programa Excel (evaluación de confiabilidad.xlsx). Como ya se dijo se aplicó la prueba piloto a 17 participantes (artistas) con cuyas respuestas se construyó una matriz de datos, a partir de la cual se construyó la hoja "Evaluación de Confiabilidad" igualmente en Excel y se procedió a realizar los cálculos para determinar la confiabilidad del instrumento mediante valor del alfa de Cronbach. Siendo el resultado final, esto es el valor de confiabilidad de $a=0.911$, lo que indica una alta estabilidad. 


\section{Tabla 1}

Coeficiente de confiablidad

\begin{tabular}{|c|c|c|}
\hline $\begin{array}{l}\text { 음 } \\
\stackrel{0}{0}\end{array}$ & $\begin{array}{l}\text { Sumatoria de las varianzas } \\
\text { de los ítems }\end{array}$ & 18.92 \\
\hline$\overline{0}$ & Número de ítems & 86 \\
\hline jo & Alfa de Crombach & 0.911 \\
\hline
\end{tabular}

Nota. Respuestas del grupo piloto.

Luego de los procesos de Validación de contenido por parte de los expertos especificados en la metodología y la prueba piloto para asegurar la confiabilidad de los ítems se obtuvo una versión definitiva de la Guía; la misma que se anexa en el presente artículo.

\section{Discusión}

Respecto de los autores que nos antecedieron en la construcción de nuestra encuesta (Ganoza 1986, Raqueta 1995, Cuenca 2006, Robalino 2006 y Arias 2009) habría que precisarse que los tales trabajaron desde sus respectivas áreas las condiciones laborales de los artistas y en especial de los docentes peruanos. Destacamos de entre ellos las propuestas de Ganoza cuya tesis es señera en la preocupación por el artista. También, resaltar los retratos que Raqueta nos ofreció de los artistas peruanos más conocidos internacionalmente, habría que incluir en esta línea cualitativa el trabajo de Ana María Echevarría (2010); antecedentes que nos estimularon para dejar abiertas algunas preguntas de la encuesta, lo que permitirá en su aplicación ahondar en las narrativas o experiencias laborales de los artistas.

Como reseñamos, el 2018 los investigadores de la Universidad de Buenos Aires publicaron, resultado de tres años continuos de investigación, un estudio sobre las condiciones laborales de los trabajadores del espectáculo en Buenos Aires (1902 1955). El enfoque asumido fue el histórico; reconociendo los mismos autores sus límites teóricos, geográficos (se centraron en la capital argentina), y temporales (primera 


\section{8}

mitad del siglo XX). Si bien consideramos propicio sumarnos a este proyecto para tener un panorama más claro de la realidad del artista latinoamericano, habría que precisar nuestras particularidades metodológicas plasmadas en el instrumento de investigación, motivo del presente artículo.

En El objeto del capital Althusser (2004) nos incita a formularnos sobre ¿̇cómo se articula la pluralidad de lo social con la unidad? Lo cual implica pensar las diferencias de los social como irreductibles. Esto es, no hay un área de lo social que sea más importante que las otras. El que la economía tenga un lugar distinto y su propia especificidad no determina la pluralidad de las otras áreas. Desde esta perspectiva, Althusser diferiría con una de las ideas marxistas más fuertes de esta corriente de pensamiento: que la economía sea la base de las otras áreas. En tal, sentido y siguiendo los postulados althusserianos no es nuestra pretensión que la presente Guía, sitúe al artista en el circuito de la producción económica. No, solamente. Y menos, que el conocimiento de dichas condiciones económicas expliquen la particularidad estética o creativa del mismo.

El conocimiento de dichas condiciones, nos debiera conducir a pensar críticamente la práctica como objeto ideológico (Althusser 1994); esto sucede cuando se toma a los objetos como dados y constituidos. Suele ser común pensar, aún entre los mismos artistas, que la tarea artística implica labores autogestivas de parte del trabajador artístico; quedando, en situaciones de crisis, ante el desamparo social y económico. En otras palabras, se trata de entender la labor de los artistas en vínculo con las otras áreas de su ser social (la educación, la salud, el ocio, lo económico, etc.) sin descuidar la especificidad de su arte: hacer arte.

\section{Conclusiones}

Las condiciones laborales de los artistas es un tópico poco explorado tanto en las ciencias sociales como en las mismas humanidades. Una de las razones que lo explican tiene que ver con la concepción del arte y del artista como ajenos al terreno productivo. 
En razón a los instrumentos sociodemográficos se ha podido construir la Guía de Encuesta; este instrumental es basto en el análisis de los sectores agrícola, educativo y de salud. Pero poco explorado en el campo artístico.

La Guía de encuesta que acompaña el presente artículo en los anexos fue sometido a evaluación de expertos considerando la complejidad del mismo por lo que se demandó la asistencia de jueces de múltiples disciplinas, pero todos ellos vinculados al arte.

Finalmente, la confiablidad de los ítems de la Guía está refrendados por la aplicación de la prueba piloto entre los artistas plásticos. Resta su aplicación en los otros sectores artísticos como las artes escénicas y musicales y las artes populares; así como en un espectro muestral más amplio.

Declaración de no tener conflictos de interés. Los autores comunicamos no tener conflictos de intereses alguno respecto del presente artículo de investigación.

\section{Referencias}

Althusser, L. (1994) Ideología y aparatos ideológicos del Estado en Ideología y aparatos ideológicos del Estado. Freud y Lacan. Buenos Aires: Nueva Visión.

Althusser, L. (2004) El objeto de El capital, en Louis Althusser y Etienne Balibar, Para leer El capital. México D. F.: Siglo XXI, 2004.

Álvarez Esteban, Ramón. (2003). Las preguntas de respuesta abierta y cerrada en los cuestionarios. Análisis estadístico de la información. Metodología de Encuestas 5(1) 45-54.

Arias Nieto, Custodio (2009) Condiciones de trabajo en el Perú del siglo XXI. Sociológica 1 (1), 209-226.

Cuenca, Ricardo (2006). Estudio de caso en Perú. En Rosalino Campos, Magaly y Körner, Antón (coordinadores) (2006) Condiciones de trabajo y salud docente. Lima: Unesco, Proeduca y GTZ. 171-194.

Decreto Supremo No 058-2004-PCM (2004) Reglamento de la Ley del artista intérprete y Ejecutante. Casa de Gobierno, Lima.

Echevarría Lübbert, Ana María (2010) Intimidad de la creación. Talleres de artistas plásticos del Perú. Lima: Scotiabank 


\section{0}

Ganoza Zapata, Myriam (1986) El contrato de trabajo del artista. Tesis para optar el grado de Bachiller en Derecho. Pucp.

Ley 28131. (2003) Ley del Artista, Interprete y Ejecutante. Congreso de la República del Perú. Lima.

Mauro, Karina (2018) Entre el mundo del arte y el mundo del trabajo. Herramientas conceptuales para comprender la dimensión laboral del trabajo artístico. Telón de fondo 27. 114-143.

Menéndez, A. (2016) Confiabilidad. Taller CES. 78-82. Recuperado de https://www.academia.edu/5894329/A._Men\%C3\%A9ndez_Taller_CES_15_Co nfiabilidad

Ministerio de Cultura del Perú (2016) Recomendación UNESCO Relativa a la Condición del Artista. Lima: Ministerio de Cultura.

Raqueta Buj, Remedios (1995) El trabajo de los artistas. Valencia: Tirant lo Blanch.

Robalino Campos, Magaly (2006) Las condiciones de trabajo y salud docente; aportes, alcances y límites del estudio. En Rosalino Campos, Magaly y Körner, Antón (coordinadores) (2006) Condiciones de trabajo y salud docente. Lima: Unesco, Proeduca y GTZ.

Varios (2018) Dossier: Condiciones laborales de los trabajadores del espectáculo en Buenos Aires (1902 - 1955). Telón de fondo 27. Dossier, producto del Proyecto UBACYT "Los trabajadores del espectáculo en Buenos Aires: la especificidad laboral como condicionamiento de su situación social, cultural y gremial (1902-1955)". Universidad de Buenos Aires, Argentina.

Yangali, J. y Ramos, A. (2006) Sinopsis de la producción de los pintores huancaínos. Informe Final. Informe de investigación docente. Universidad Nacional del Centro del Perú.

Yangali, J. (2019) Investigación desde y por las artes en el ambiente universitario peruano, caso Junín. Karina Mauro. Artes y producción de conocimiento. Experiencias de integración de las artes en la universidad. Buenos Aires/ Los Ángeles: Argus-a. 


\section{Anexo 1}

\section{Guía de encuesta}

\section{Investigación: condiciones laborales de los artistas}

El objetivo de la presente guía de encuesta es conocer las condiciones laborales del artista en Junín, es una ficha semiestructurada que recoge datos cerrados y abiertos de los participantes en el estudio. Le solicitamos nos brinde la información completando los datos de la siguiente ficha o respondiendo a las siguientes inquietudes:

\section{DATOS GENERALES}

Nombres y apellidos:

Estado civil: soltero $\square$ casado $\square$ viudo $\square$ divorciado $\square$

Labor(es) artística(s) de su especialidad:

Artes visuales

Artes escénicas (incluye música)

Artes populares

Condición de dependencia económica de usted como artista.

Independiente

De sus padres, conyugue o familiares $\square$

De sus hijos

Otros

Especificar:

Número de hijos o familiares (padres)

Dependientes económicamente de usted:

Independientes económicamente de usted:

\section{FORMACIÓN VOCACIONAL Y ACADÉMICA}

Título(s) y Grados

En arte:

Otros:

Conocimiento y dominio de idiomas, aparte del español (detalle):

Quechua $\square \quad$ Inglés $\square \quad$ Portugués $\square \quad$ Otro:

Detalle su conocimiento y dominio de informática $u$ otras competencias técnicas:

Motivación para dedicarse al arte:

Propia: 
292

Por estimulo de otros (padres, hijos, profesores, etc.)

Necesidad (para con los hijos, la esposa, autorealización)

Deseos de realización (truncas o no) ¿qué más quiso o quisiera realizar?:

Otras labores artísticas:

Otras ocupaciones:

\section{CONDICIONES DE SALUD}

Cuenta con seguro social (pensión de/para jubilación)

$$
\mathrm{Si} \square \mathrm{No} \square
$$

Cuenta con seguro médico

Si $\square \mathrm{No} \square$

Cuenta con seguro contra-accidentes (invalidez y muerte)

Si $\square$ No $\square$

Cuenta con seguro de protección a la propiedad intelectual

$\mathrm{Si} \square \mathrm{No} \square$

¿Quién le proporcionó o cómo adquirió estos seguros?

Entidad vinculada al arte

Entidad no vinculada al arte

Por su propia cuenta

\section{Exigencias ergonómicas}

¿Cuál o cuáles son las exigencias ergonómicas que le demanda su trabajo vinculado al arte?

Estar de pie

Forzar la voz

Trabajar en temperaturas inadecuadas

Contaminación acústica

Contaminación medio ambiental

Otros

Especificar:

\section{Perfil patológico tanto de enfermedades como de malestares percibidos}

¿Cuál o cuáles de estos malestares ha sentido a causa de su trabajo vinculado al arte?

Estrés

Depresión

Neurosis 
Enfermedades psicosomáticas como gastritis $\square$

Trastornos en su régimen nutricional (dieta)

Temor por las condiciones laborales de riesgo elevado y/o frecuente $\square$

Físicos Especificar:

Especifique si tiene problemas con consumo de alguna de las siguientes sustancias psicoactivas

Alcohol

Tabaco

Marihuana

Otro

$\square$ Especifique:

Recibe tratamiento y atención médica respecto de sus enfermedades o adicciones

$\mathrm{Si} \square \quad$ No

Frecuencia:

Diaria $\square \quad$ Semanal $\square \quad$ Mensual $\square \quad$ Según cita médica

\section{CONDICIONES LABORALES}

Remuneración actual (promedio):

Por su labor artística:

Por su labor no artística:

Condiciones de estabilidad laboral artística o no:

Nombrado

Contratado

Régimen laboral

En Arte: Público $\square$ Privado

Otro: Público $\square$ Privado

Proyecto para jubilación (en trabajo artístico)

Régimen estatal (ONP)

Régimen privado (AFP)

Régimen familiar/personal (Ahorros)

Aún no lo ha pensado

Jornada laboral en Arte (diaria/semanal)

Tiempo de trabajo

Horas al día:

Días a la semana:

Tiempo de descanso: 


\section{4}

Horas al día:

Días a la semana:

¿A qué distancia de su domicilio queda su centro de trabajo artístico? (km):

Detalle en minutos el tiempo que le toma trasladarse hacia este lugar:

Para trasladarse a su centro de trabajo artístico utiliza:

Transporte público

Movilidad propia

Caminando

Otros

Especificar:

A qué distancia de su domicilio queda su centro de trabajo no artístico $(\mathrm{km})$ :

Detalle en minutos el tiempo que le toma trasladarse hacia este otro lugar:

Tiempo dedicado a las siguientes actividades vinculadas al trabajo en arte (diaria/semanal o Mensual/anual)

Perfeccionamiento (cursos, posgrados)

Días a la semana:

Semanas al mes:

Meses al año:

Preparación de materiales

Días a la semana:

Semanas al mes:

Meses al año:

Investigación

Días a la semana:

Semanas al mes:

Meses al año:

Labor artística propiamente dicha (creación)

Días a la semana:

Semanas al mes:

Meses al año:

Participación en eventos académicos o artísticos (internacionales, nacionales, locales)

Días a la semana:

Semanas al mes:

Meses al año: 
Acciones en su "Tiempo libre" (entiéndase como no laboral) destacan algunas actividades como (incluya todas las que realiza):

Realizar ejercicios (deporte)

Frecuentar amigos

Pasar con la familia

Mascotas o plantas

Leer sin fines laborales

Realizar actividades comunitarias o eclesiales

Asistir a actividades culturales

Ver vídeos u oír música (en solitario o en familia)

Estudiar temas de su interés (cursillos virtuales, presenciales, a distancia)

Viajes (locales, nacionales, internacionales) vacacionales

\section{CONDICIONES MATERIALES DE TRABAJO ARTÍSTICO}

Infraestructura laboral, su trabajo artístico lo realiza en:

Vivienda (domicilio)

Aire Libre

Taller/Estudio/Auditorio/Galería

El ambiente donde desarrolla su labor artística es:

Propio $\square$ Alquilado $\square$ Prestado $\square$ Donado

La iluminación de este ambiente, en relación a su desempeño artístico es:

Adecuada $\square$ Inadecuada

La ventilación y temperatura de este ambiente, en relación a su desempeño artístico es:

Adecuadas $\square \quad$ Inadecuadas

La comodidad de este ambiente, en relación a su desempeño artístico es:

Adecuada $\square$ Inadecuada

Este ambiente presta medidas de seguridad en casos de:

Incendios

Inundaciones

Movimientos telúricos

Para la edad o salud (rampas, pasamanos, etc.)

Otros

Especificar:

La adquisición de insumos, materiales, equipos, vestido (para el trabajo), etc. Los obtiene con:

Recursos propios $\square$ Donación $\square$ Provisión del empleador 


\section{6}

Para realizar su labor artística cuenta con personal de apoyo (sonidista, iluminación, transporte, etc.) por parte de un:

Familiar

Integrante de la compañía o colectivo o agrupación

Personal contratado a tiempo parcial o completo o para tarea específica

¿Quién asume los costos del personal de apoyo?

$$
\text { El empleador o proyecto } \square \text { El mismo artista } \square
$$

¿Quiénes o qué instituciones suelen financiar/adquirir su producción artística?

Organización laboral

Solo si forma parte de alguna organización de artistas diga a cuál:

Asociación

Movimiento

Sindicato

Colectivo

Otras asociaciones:
Detalle:

Detalle:

Detalle:

Detalle:

Detalle:

¿Su participación en estas organizaciones es?:

Activa $\square$ Inactiva

Simpatizante Comprometida

Conoce y exige reconocimiento económico por derechos autorales de:

Exhibición $\quad \mathrm{Si} \square \mathrm{No} \square$

Difusión $\quad \mathrm{Si} \square$ No $\square$

Traducción $\quad \mathrm{Si} \square \mathrm{No} \square$

Virtualización Si $\square$ No $\square$

Mantiene vínculo con "empleadores" o promotores culturales (salas, publicidad, curación, etc.) como:
Ministerio de Cultura
Si $\square$ No $\square$
Institutos o Casas culturales municipales
Si $\square$ No $\square$
Detalle:
Centros culturales (institucionales):
Si $\square$ No $\square$
Detalle:

¿Cuáles son los condicionamientos o contraprestaciones por el apoyo brindado de los anteriores?

Realizar la actividad gratuita

Ingresos (si se cobra) compartidos

$\square$, especificar porcentajes:

Publicidad o promoción de la entidad auspiciadora 
Responsabilidad social de la entidad

Exclusividad o estreno

Cesión de derechos o donación de una obra

\section{EXPERIENCIAS LABORALES}

Ha realizado trabajo compulsivo (hasta acabar) por iniciativa:

Propia

Por encargo privado o público

Para un concurso

En este punto solicitamos a usted nos narre alguna de las experiencias laborales sugeridas en las siguientes líneas:

a. Experiencia de despido (en trabajo artístico):

b. Experiencia de sanción (administrativa, judicial, penal, etc.) en trabajo artístico:

c. Experiencia de discriminación salarial por género, condición social en el terreno artístico:

d. Experiencia de ascenso en la carrera artística y cultural:

e. Experiencia grata o ingrata de trabajo artístico (contrata) para el Estado:

\section{ACTITUD FRENTE AL TRABAJO ARTÍSTICO}

Defina con sus propias palabras las siguientes categorías

a. Arte:

b. Artista:

c. Trabajo artístico:

En síntesis, ¿̇Se siente satisfecho con su trabajo artístico? ¿por qué?) 


\section{8}

\section{Anexo 2}

Carta de Consentimiento informado:

Fecha

Yo

artista, acepto voluntariamente participar en

la prueba piloto de la investigación "Construcción de un instrumento para conocer las condiciones laborales de los artistas peruanos", conducida por el Profesor Jorge Luis Yangali Vargas, investigador de la Universidad Nacional del Centro del Perú.

He sido informado(a) de los objetivos, alcance y resultados esperados de este estudio y de las características de mi participación. Reconozco que la información que provea en la encuesta de esta investigación es confidencial. Además, esta no será usada para ningún otro propósito fuera de los de este estudio.

He sido informado(a) de que puedo hacer preguntas sobre el proyecto en cualquier momento y que puedo decidir que no se incluya mi participación en el informe, sin tener que dar explicaciones ni sufrir consecuencia alguna por tal decisión.

De tener preguntas sobre mi participación en este estudio, puedo contactar al Dr. Ciro Espinoza, Director del Instituto General de Investigación de la Universidad Nacional del Centro del Perú (investigacion@uncp.edu.pe) y/o al Comité de Ética de Investigación de la UNCP. Entiendo que una copia de este documento de consentimiento me será entregada, y que puedo pedir información sobre los resultados de este estudio cuando éste haya concluido (2020). Para esto, puedo contactar al Investigador Responsable del proyecto al correo electrónico jyangali@uncp.edu.pe, o al teléfono 944897382.

Nombre y firma del participante

Investigador Responsable 